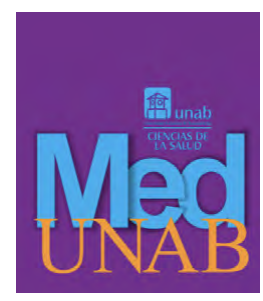

REVISTA DE LA FACULTAD

DE CIENCIAS DE LA SALUD

\title{
Perfil de resistencia antimicrobiana de aislamientos bacterianos en pacientes con infección urinaria de un centro de referencia en Bucaramanga
}

Antimicrobial Resistance Profiles of Bacterial Isolates in Patients with Urinary Tract Infections in a Reference Center in Bucaramanga

Perfil de resistência antimicrobiana de isulados bacterianos em pacientes com infecção urinária de um centro de referência em Bucaramanga

Josué Delgado-Serrano, MD. ${ }^{1}$ iD,

María José Albarracín Ruiz, MD., Sp. ${ }^{2}$ iD, Jully Andrea Rangel-Vera, MD. ${ }^{3}$ iD, Erika Galeano-Salazar, MD. ${ }^{4}$ (D),
Daniela Niño-Vargas, $M D .5$ iD, María Angélica Wilches-Cuadros, MD. ${ }^{6}$ (D), Laura Domínguez-García, MD. ${ }^{7}$ iD, Diego Torres-Dueñas, MD. MSc. $P h D .^{8}$ (D),

1. Médico. Universidad Autónoma de Bucaramanga, Santander, Colombia.

2. Médica, Especialista en Epidemiología. Secretaría de Salud de Santander, Bucaramanga, Santander, Colombia.

3. Médica. Spectrum Healthcare, Toronto, Canadá.

4. Médica. Hospital Emiro Quintero Cañizares, Ocaña, Norte de Santander, Colombia.

5. Médica, Residente de Urología. Fundación Universitaria de Ciencias de la Salud, Bogotá, Colombia.

6. Médica, Residente de Pediatría. Universidad del Rosario, Bogotá, Colombia.

7. Médica. Asociación de Profesionales de Barrancabermeja, Santander, Colombia.

8. Médico, Magíster en Farmacología, Doctor en Farmacología. Facultad de Ciencias de la Salud, Universidad Autónoma de Bucaramanga, Santander, Colombia.

Correspondencia. Diego Torres-Dueñas. Universidad Autónoma de Bucaramanga, Campus el Bosque. Email. dtorres@unab.edu.co.

\section{INFORMACIÓN DEL ARTÍCULO:}

Artículo recibido: 16 de Junio de 2020

Artículo aceptado: 07 de Septiembre de 2020

DOI: https://doi.org/10.29375/01237047.3950

Cómo citar. Delgado-Serrano J, Albarracín MJ, Rangel-Vera JA, GaleanoSalazar E, Niño-Vargas D, Wilches-Cuadros MA, Et al. Perfil de resistencia antimicrobiana de aislamientos bacterianos en pacientes con infección urinaria de un centro de referencia en Bucaramanga. MedUNAB. 2020;23(3):405-413. Doi: https://doi.org/10.29375/01237047.3950

\section{RESUMEN}

Introducción. Dentro de las infecciones bacterianas que afectan al ser humano, la infección de las vías urinarias es una de las más frecuentes, afectando hasta 
150 millones de personas en el mundo cada año. El uso indiscriminado de antibióticos y su manejo inadecuado han generado un aumento en la resistencia antimicrobiana. El objetivo del presente estudio es describir patrones fenotípicos de resistencia antimicrobiana de los microorganismos más frecuentes en pacientes con diagnóstico de infección de vías urinarias en centro de referencia de Bucaramanga. Metodología. Estudio de corte transversal, muestreo no probabilístico en pacientes con sospecha o diagnóstico de infecciones de vías urinarias que requirieron manejo intrahospitalario en una institución de salud de alta complejidad durante julio del 2017 y abril del 2018, de quienes se obtuvo urocultivo y antibiograma. Resultados. De 120 pacientes incluidos, se obtuvo un reporte de 116 urocultivos. El microorganismo más frecuente fue Escherichia coli, seguido de Klebsiella pneumoniae. El primero presentó alta sensibilidad a carbapenémicos y aminoglucósidos, baja sensibilidad a la Ceftriaxona y a la Ampicilina/Sulbactam. Por otro lado, el microorganismo Klebsiella pneumoniae presentó alta sensibilidad a carbapenémicos, pero resistencia elevada a la Ampicilina/Sulbactam y a la Ceftriaxona. El antibiótico empírico más utilizado fue la Ceftriaxona. Discusión. La distribución de microorganismos aislados es comparable con la ya descrita a nivel nacional e internacional. Los perfiles de resistencia antimicrobiana tienen puntos comunes como la resistencia a los betalactámicos, pero difieren en algunos aspectos, como la resistencia a cefalosporinas y quinolonas. Conclusiones. Los microorganismos aislados presentan altas tasas de resistencia a los betalactámicos y a las cefalosporinas, las cuales son ampliamente utilizadas. Esta información debe orientar el desarrollo de protocolos que permitan optimizar la toma de decisiones clínicas con respecto a la terapia antibiótica.

Palabras clave:

Infecciones urinarias; Infecciones bacterianas; Antimicrobianos; Resistencia antimicrobiana; Pruebas de sensibilidad microbiana.

\begin{abstract}
Introduction. Among the bacterial infections that affect human beings, urinary tract infections are one of the most common, affecting up to 150 million people worldwide each year. Indiscriminate use of antibiotics and their improper handling has generated an increase in microbial resistance. This study's objective is to describe the phenotypic patterns of antimicrobial resistance of the most frequent microorganisms in patients with urinary tract infection diagnoses in a reference center in Bucaramanga. Methodology. A cross-sectional study with non probability sampling on patients with suspected or diagnosed urinary tract infections who required hospital treatment at a high-complexity health institution during July, 2017 and April, 2018, from whom urine cultures and antibiograms were obtained. Results. Out of 120 patients, a report was obtained for 116 urine cultures. The most frequent microorganism was Escherichia coli, followed by Klebsiella pneumoniae. The former presented high sensitivity to carbapenem antibiotics and aminoglycosides and low sensitivity to Ceftriaxone and Ampicillin/ Sulbactam. On the other hand, the microorganism Klebsiella pneumoniae presented high sensitivity to carbapenem antibiotics, but elevated resistance to Ceftriaxone and Ampicillin/Sulbactam. The empiric antibiotic used most was Ceftriaxone. Discussion. The distribution of isolated microorganisms is comparable to that which has been described nationally and internationally. Antimicrobial resistance profiles have points in common, such as resistance to beta- lactams, but differ in some aspects, such as resistance to cephalosporins and quinolones. Conclusions. Isolated microorganisms present high rates of resistance to beta-lactams and cephalosporins, which are widely used. This information must guide developing protocols that allow optimizing clinical decision- making with respect to antibiotic therapy.
\end{abstract}

Keywords:

Urinary tract infections; Bacterial infection; Antimicrobials; Antimicrobial resistance; Microbial Sensitivity Tests.

\title{
RESUMO
}

Introdução. Dentre as infecções bacterianas que afetam o homem, a infecção do trato urinário é uma das mais frequentes, afetando até 150 milhões de pessoas no mundo a cada ano. O uso indiscriminado de antibióticos e seu manejo inadequado têm gerado aumento da resistência antimicrobiana. O objetivo 
deste estudo é descrever padrões fenotípicos de resistência antimicrobiana dos microrganismos mais frequentes em pacientes com diagnóstico de infecção do trato urinário em um centro de referência em Bucaramanga. Métodos. Estudo transversal, com amostra não probabilística, em pacientes com suspeita ou diagnóstico de ITU que precisaram de manejo intra-hospitalar em uma instituição de saúde de alta complexidade no período de julho de 2017 a abril de 2018. Foram obtidos urocultura e antibiograma desses pacientes. Resultados. De 120 pacientes incluídos, foi obtido um relatório de 116 uroculturas. O microrganismo mais frequente foi Escherichia coli, seguido por Klebsiella pneumoniae. O primeiro apresentou alta sensibilidade a carbapenêmicos e aminoglicosídeos e baixa sensibilidade a Ceftriaxona e a Ampicilina/Sulbactam. Por outro lado, o microrganismo Klebsiella pneumoniae apresentou alta sensibilidade a carbapenêmicos, mas alta resistência a Ampicilina/ Sulbactam e a Ceftriaxona. O antibiótico empírico mais amplamente utilizado foi a Ceftriaxona. Discussão. A distribuição dos microrganismos isolados é comparável àquela já descrita a nível nacional e internacional. Os perfis de resistência antimicrobiana têm pontos comuns como resistência aos beta-lactâmicos, mas diferem em alguns aspectos, como resistência às cefalosporinas e quinolonas. Conclusão. Microrganismos isolados apresentam altas taxas de resistência aos beta-lactâmicos e cefalosporinas, que são amplamente utilizados. Esta informação deve nortear o desenvolvimento de protocolos que permitam otimizar a tomada de decisão clínica em relação à antibioticoterapia.

Palavras-chave:

Infecções urinárias; Infecções bacterianas; Anti-infecciosos; Resistência antimicrobiana; Testes de sensibilidade microbiana.

\section{Introducción}

Las infecciones de vías urinarias (IVU) comprenden un conjunto de entidades clínicas que son comunes tanto en el escenario ambulatorio como en el hospitalario (1). Actualmente se encuentran entre las infecciones más frecuentes, afectando hasta 150 millones de personas en el mundo cada año (2). Se ha estimado que entre un $40 \%$ y un $50 \%$ de las mujeres tendrá un episodio de infección urinaria en su vida, mientras que en hombres los reportes de infección son inferiores al $12 \%$, con cultivos positivos apenas de un $5 \%$ en esta población $(3,4)$. Las IVU se pueden presentar clínicamente como Cistitis (infección de la vejiga) o Pielonefritis (infección del riñón), y el microorganismo más frecuentemente involucrado es la Escherichia coli uropatogénica, que es responsable del $75 \%$ al $95 \%$ de las IVU, seguido de la Klebsiella pneumoniae (2,5). En Colombia, varios estudios locales y reportes de vigilancia epidemiológica han buscado establecer la prevalencia y los factores de riesgo poblacionales, así como la caracterización de los microorganismos aislados y un acercamiento a sus perfiles de resistencia y sensibilidad, encontrando alta prevalencia de la Escherichia coli y la Klebsiella pneumoniae (6-12), siendo la E. coli el primer agente infeccioso causante de IVU a nivel nacional. Esto se determinó en un estudio que incluyó a más de 30,000 urocultivos (13).

Un problema creciente es el aumento de la resistencia bacteriana que ha reducido las opciones de tratamientos empíricos (5). Esto se debe a diversos factores, como el uso irracional de antibióticos y la falta de adherencia al tratamiento (14), y representan un alto riesgo de fracaso terapéutico, especialmente en pacientes susceptibles de contraer estos gérmenes, como es el caso de las personas con alguna enfermedad renal crónica, uso previo de antibióticos, hospitalización reciente, pielonefritis o diabetes (15). La resistencia bacteriana en IVU ha aumentado y varía mucho dependiendo de cada región (16), pero las tendencias globales, nacionales y locales de resistencia de las bacterias a los antibióticos de uso tradicional como la Ampicilina, la Ciprofloxacina o el Trimetropim-Sulfametoxazol, han convertido a las infecciones urinarias en un reto para el personal de salud $(13,17)$, tanto en la población general como en diferentes grupos de pacientes. Este es el caso de las personas con diagnóstico de diabetes mellitus, en las que se ha reportado alta prevalencia de cepas productoras de betalactamasas de espectro extendido y de carbapenemasas (18). También es el caso de las pacientes gestantes, en quienes se han reportado enterobacterias resistentes a cefalosporinas de tercera generación (19).

El aumento en las tasas de resistencia antimicrobiana representa un problema de salud pública, ya que puede comprometer la efectividad de la terapia empírica y aumentar las tasas de recurrencia, estancia hospitalaria y muerte $(7,8)$. Por lo tanto, son necesarias la identificación y la actualización de perfiles de resistencia locales para la optimización de la terapia antimicrobiana. 
En la actualidad, la información sobre estos perfiles en Bucaramanga es escasa, por lo que se plantea este estudio con el objetivo de describir los patrones de resistencia a antibióticos de los microorganismos aislados en pacientes con diagnóstico de infección urinaria en un centro de referencia de la ciudad.

\section{Metodología}

Se realizó un estudio de corte transversal, a partir de un estudio longitudinal analítico, en el que se incluyeron, mediante muestreo no probabilístico por conveniencia, a todos los pacientes mayores de edad atendidos por urgencias que tuvieran sospecha o diagnóstico de infección de vías urinarias y que requirieron manejo intrahospitalario, en una institución prestadora de salud de alto nivel de complejidad durante el periodo comprendido entre julio del 2017 y abril del 2018. Se excluyeron del estudio las pacientes en estado de gestación y población pediátrica.

Para la recolección de información se diseñó un formato elaborado por el equipo investigador con el apoyo de profesionales con experiencia en enfermedades infecciosas. Este formato fue diligenciado por un entrevistador capacitado, con base en la entrevista directa al paciente y la posterior revisión de su historia clínica. Además de datos sociodemográficos, antecedentes, presentación clínica y resultados paraclínicos de muestra sanguínea, este formato incluyó el reporte de urocultivo y su respectivo antibiograma con reporte de sensibilidad, cuando estuviera disponible.

Los antibiogramas fueron procesados por método automatizado con equipos Vitek 2 XL de la casa comercial Biomerieux, con puntos de corte de concentración inhibitoria mínima (MIC, por sus siglas en inglés) de acuerdo con el estándar del Instituto de Estándares Clínicos y de laboratorio (CLSI). Para el análisis descriptivo se tuvieron en cuenta las categorías Sensible, Intermedio y Resistente.

En el procesamiento de los datos se construyó una base de datos en Excel con base en la información consignada en el formato físico de captación de pacientes. La información fue registrada por dos estudiantes de medicina capacitados, en dos archivos independientes, y luego verificada por uno de los investigadores para dejar un único documento.

Para el análisis estadístico se realizó un análisis univariado. Las variables cualitativas se describieron por medio de frecuencias con su respectivo porcentaje y proporciones; mientras las variables cuantitativas se describieron con medidas de tendencia central, de acuerdo con el tipo de distribución de la muestra.

Este estudio fue aprobado por el Comité de Ética de la Universidad Autónoma de Bucaramanga, y de la Fundación oftalmológica de Santander Clínica Carlos Ardila LulleFOSCAL, y se realizó teniendo en cuenta la declaración de Helsinki, así como la Resolución 8430 de 1993, según la cual se clasifica como una investigación sin riesgo, puesto que los registros de laboratorio se obtuvieron de la historia clínica. Adicionalmente, se obtuvo consentimiento informado escrito de cada paciente.

\section{Resultados}

Se incluyeron 120 pacientes con diagnóstico de IVU atendidos en el servicio de urgencias de la institución. El grupo tenía una edad promedio 65 años y era predominantemente femenino. De estos pacientes, la mayoría tenía antecedente de infección urinaria, y la comorbilidad sistémica más frecuente fue la hipertensión arterial, entre otras como la diabetes mellitus y la enfermedad renal crónica. Las características demográficas y clínicas se describen en la tabla 1.

Tabla 1. Características demográficas y clínicas de la población estudiada

\begin{tabular}{llc}
$\begin{array}{l}\text { Características de la } \\
\text { población }\end{array}$ & Número (\%) & IC (95\%) \\
$\begin{array}{l}\text { Sexo } \\
\text { Hombre }\end{array}$ & $47(39,17 \%)$ & $30,74-48,28$ \\
\hline $\begin{array}{l}\text { Mujer } \\
\text { IVU previa }\end{array}$ & $63(60,83 \%)$ & $51,71-69,25$ \\
\hline $\begin{array}{l}\text { Uso previo } \\
\text { antimicrobiano }\end{array}$ & $51(42,86 \%)$ & $34,16-52,01$ \\
\hline Comorbilidades asociadas & & $47,98-65,83$ \\
\hline Diabetes mellitus & $34(28,81 \%)$ & $(21,26-37,74)$ \\
\hline Enfermedad renal crónica & $22(18,64 \%)$ & $12,52-26,83$ \\
\hline Hipertensión arterial & $58(49,15 \%)$ & $40,12-58,23$ \\
\hline Enfermedad oncológica & $17(14,53 \%)$ & $9,16-22,26$ \\
\hline
\end{tabular}

Fuente: elaboración propia.

Del grupo completo, a 4 pacientes no se les realizó antibiograma por tratarse de IVU no complicada. Durante la estancia hospitalaria, se obtuvo un reporte de 116 urocultivos, de los cuales 95 resultaron positivos $(81.90 \%)$. El microorganismo más frecuentemente aislado 
fue Escherichia coli $(\mathrm{n}=79)$ seguido por Klebsiella pneumoniae $(\mathrm{n}=14)$. También se obtuvo aislamiento de Proteus spp y de Enterobacter cloacae en un mínimo porcentaje (figura 1).

Figura 1. Microorganismos aislados durante la estancia hospitalaria de pacientes con infección urinaria.

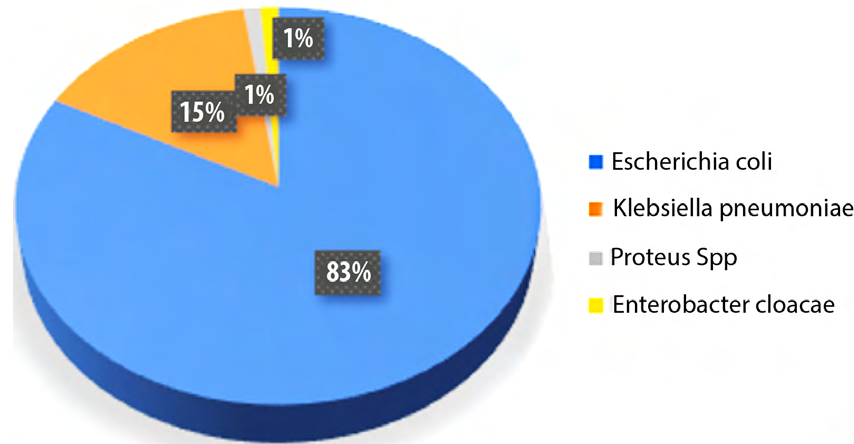

Fuente: elaboración propia.

En la tabla 2 se observan los medicamentos instaurados en Urgencias. El medicamento antibiótico más usado fue la Ceftriaxona, presente en el $68.4 \%$ de los casos, seguido por el Ertapenem, con una frecuencia del 8.6\%.

Tabla 2. Manejo empírico de antibiótico

\begin{tabular}{lcc}
$\begin{array}{l}\text { Antibiótico empírico } \\
\text { iniciado }\end{array}$ & $\mathbf{n : ~ 1 1 6 ( \% )}$ & IC \\
\hline Ceftriaxona & $79(68.1 \%)$ & $(58.95-76.03)$ \\
\hline Piperacilina-Tazobactam & $7(6 \%)$ & $(2.87-12.24)$ \\
\hline Meropenem & $4(3.4 \%)$ & $(1.28-8.94)$ \\
\hline Amikacina & $3(2.5 \%)$ & $(0.82-7.83)$ \\
\hline Ciprofloxacino & $2(1.7 \%)$ & $(0.42-6.76)$ \\
\hline Cefoxitin & $2(1.7 \%)$ & $(0.42-6.76)$ \\
\hline Cefazolina & $2(1.7 \%)$ & $(0.42-6.76)$ \\
\hline Ertapenem & $10(8.6 \%)$ & $(4.65-15.41)$ \\
\hline Gentamicina & $1(0.8 \%)$ & $(0.11-6.02)$ \\
\hline Cefotetan & $1(0.8 \%)$ & $(0.11-6.02)$ \\
Cefotetan & $1(0.8 \%)$ & $(0.11-6.02)$ \\
\hline Cefepime & $1(0.8 \%)$ & $(0.11-6.02)$ \\
\hline Cefalotina & $1(0.8 \%)$ & $(0.11-6.02)$ \\
\hline Ampicilina & $1(0.8 \%)$ & $(0.11-6.02)$
\end{tabular}

Fuente: elaboración propia.
Por otra parte, se analizaron los perfiles de susceptibilidad antimicrobiana de los gérmenes más frecuentemente encontrados. En la figura 2 se observa el perfil de resistencia a antimicrobianos de Escherichia coli, y se reagrupan los antibióticos tamizados en tres categorías: Sensible, Intermedio y Resistente. Se encontró que el grupo de antibióticos carbapenémicos tuvo el mayor perfil de sensibilidad con una resistencia menor al $5 \%$, resaltando al Imipenem, en el cual no se registró resistencia. Por otra parte, el antibiótico al que se observó mayor resistencia fue la Ampicilina/Sulbactam, con un $50.8 \%$ de microorganismos resistentes y un $16.4 \%$ de ellos con sensibilidad intermedia.

Figura 2. Perfil de sensibilidad a antimicrobianos de Escherichia coli en aislamientos microbiológicos de los pacientes con infección urinaria.

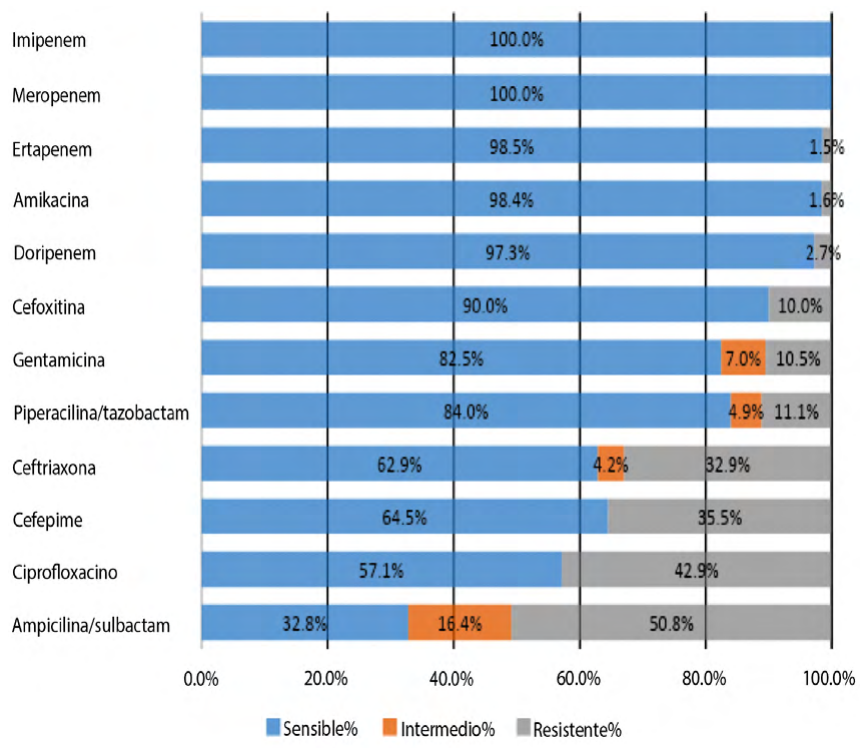

Fuente: elaboración propia.

Por su parte, en la figura 3 se observa el perfil de resistencia antibiótico del segundo germen más frecuente, Klebsiella pneumoniae. Se encuentra que el antibiótico con mejor perfil fue el Doripenem con una sensibilidad del 90.9\% $(n=13)$. Por el contrario, se encontraron dos antibióticos con la mayor resistencia antimicrobiana, la Ampicilina/ Sulbactam y la Ceftriaxona, con una resistencia del $68.8 \%$. Para este microorganismo no se registraron reportes de antibiograma con sensibilidad intermedia a ningún medicamento. 
Figura 3. Perfil de sensibilidad a antimicrobianos de la Klebsiella pneumoniae en infección urinaria.

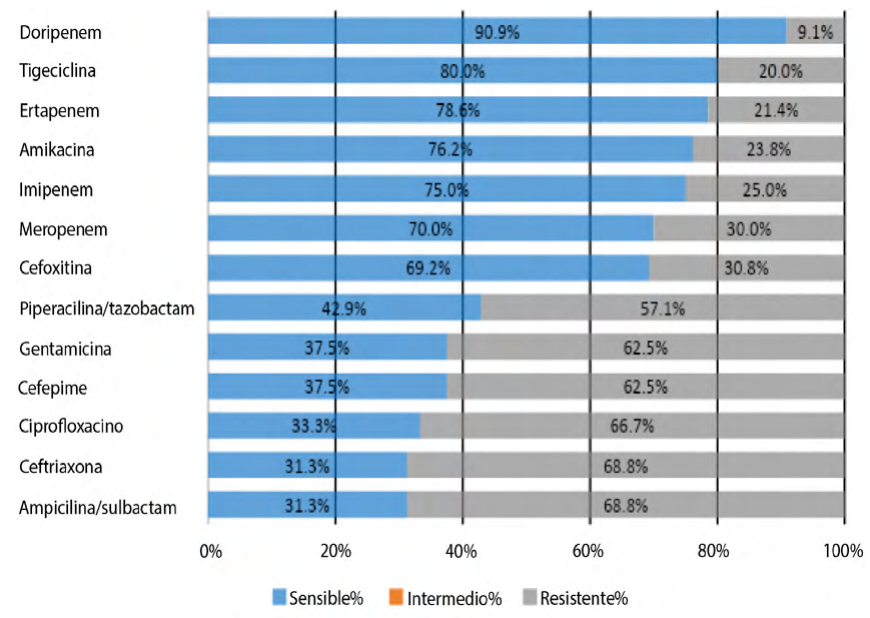

Fuente: elaboración propia.

\section{Discusión}

Este estudio se planteó con el objetivo de realizar una primera aproximación los patrones fenotípicos de resistencia antimicrobiana de los microorganismos más frecuentemente aislados en pacientes con diagnóstico de IVU en un centro de referencia de la ciudad de Bucaramanga. Así, con respecto a los microorganismos aislados, este trabajo encontró una distribución similar a la reportada previamente por numerosos estudios internacionales, en los cuales se observa que el germen más prevalente es Escherichia coli, seguido por especies de Klebsiella, dentro de las cuales la más frecuente es Klebsiella pneumoniae (20-22). De la misma manera, esta distribución de microorganismos se ha reportado en diversos estudios del contexto nacional (23-25), de especial relevancia el trabajo desarrollado por Pardo y colaboradores (13), quienes realizaron seguimiento por 5 años a más de 30 mil pacientes, y reportaron prevalencia de E coli superiores al 70\% y de K. pneumoniae cercanas al $10 \%$.

Por su parte, en un estudio realizado en Cartagena, los principales agentes etiológicos fueron: E. coli (46.7\%), E. coli betalactamasas de espectro extendido (BLEE) (17.9\%) y Pseudomona aeruginosa (10.85\%) asociado a población en condiciones de alta vulnerabilidad (11). En otro estudio realizado en Bogotá se reportó aislamiento de E. coli (62.58\%), Enterococcus faecalis (12.33\%), Proteus spp. (8.74\%) y Klebsiella pneumoniae (6.83\%) (10).

De lo anterior se resalta que las bacterias gramnegativas siguen siendo protagonistas en las infecciones urinarias y que Klebsiella pneumoniae ha cobrado importancia, dada su creciente prevalencia como agente etiológico, como se reporta en el trabajo de Pardo y colaboradores, en el que se evidencia que las especies de Proteus han cedido el segundo lugar (13), lo que debe ser tenido en cuenta para la elección del tratamiento empírico. A pesar de lo anterior, es importante resaltar que, en los protocolos de manejo recientes, el manejo empírico se basa principalmente en el perfil de sensibilidad de Escherichia coli $(26,27)$.

Con respecto a los patrones fenotípicos de resistencia de los microorganismos más frecuentes, para el caso de E. coli, este estudio resalta la alta sensibilidad a carbapenémicos y aminoglucósidos. Sin embargo, es importante aclarar que estos antibióticos no son de primera elección, dado su amplio espectro de acción, que puede llevar a presión selectiva y mayor resistencia, especialmente en el caso de los carbapenémicos $(26,28,29)$, así como al perfil de seguridad de los aminoglucósidos, que deben ser utilizados con precaución en pacientes con enfermedad renal y su uso se debería reservarse para pacientes no críticos con IVU no complicada $(28,30)$.

Los anteriores hallazgos son similares a los reportados en otros trabajos: en un estudio prospectivo multicéntrico internacional en el que se incluyeron mujeres con IVU no complicada, el $E$. coli mostró baja sensibilidad a la Ampicilina (48.3\%), Trimethoprim/Sulfametoxazol (29.4\%) y al ácido nalidíxico (18.6\%), mientras que otros medicamentos como el Cefuroxime y la Ciprofloxacina, con resistencias inferiores al 10\% mostraron ser más activos contra las cepas aisladas (31). Lo anterior se corresponde también con lo reportado en una revisión de la literatura que resume la evidencia epidemiológica de las IVU, encontrando en el perfil de resistencia de la Coli entre los años 2003 a 2006 que la Ampicilina obtuvo la mayor tasa de resistencia, superando el $60 \%$ en la mayoría de reportes (32). También, en un estudio transversal realizado en 2 hospitales durante el 2014, se encontró que para E.coli la sensibilidad fue reducida para la Ampicilina-Sulbactam con una sensibilidad del $47 \%$, resaltándose que la mayor sensibilidad antibiótica fue frente al Meropenem con un $87 \%$, seguido por la Piperacilina Tazobactam con un $83 \%$ (33), con la salvedad, nuevamente, de tener en cuenta que estos antibióticos se deben reservarpara el paciente críticamente enfermo, y no son de primera línea según la mayoría de posiciones científicas $(26,28)$. Para el caso colombiano, se ha registrado que $E$. Coli muestra resistencias a Ceftriaxona cercanas al $38 \%$, a Ampicilina cercanas al $75 \%$ y a Amoxicilina/Clavulanato cercanas al $95 \%$ en salas de hospitalización general en Bogotá, Antioquia y Valle del Cauca (12); así como resistencias del 54\% a la Ampicilina, 39\% a Trimetropim-Sulfametoxazol y del 28\% a la Ciprofloxacina (13), distribución similar a la reportada en el presente estudio. 
cepas de E. coli productoras de beta-lactamasas de

Para Klebsiella pneumoniae, este estudio mostró que la mayor sensibilidad se registró frente a carbapenémicos, mientras que otros antibióticos de uso común, como la Ampicilina/Sulbactam y la Ceftriaxona mostraron resistencias elevadas. Este perfil de resistencia se compara con el reportado en un estudio chino en el cual $K$. penumoniae mostró una mayor resistencia a Cefazolina (35.1\%) y Ampicilina/Sulbactam (32.6\%), y una menor resistencia a Ertapenem $(2.7 \%)(34)$. Otro estudio realizado en Marruecos reportó aislamiento de $K$. pneumoniae en el $22 \%$ de las muestras de orina, con resistencia al Trimetoprim-Sulfametoxazol en un $61 \%$, seguido por Amoxicilina/Clavulanato con una tasa del 51\% (35).

A nivel nacional, se encontró que la mayor resistencia encontrada para este germen fue frente a la Ampicilina con resistencia de hasta el $85 \%$, seguido por Ampicilina Sulbactam (hasta $43 \%$ ) y Ceftriaxona con resistencia de alrededor del $30 \%$, con bajas resistencias a la Ciprofloxacina $(12,13)$, lo que también se asemeja al presente estudio. Cabe resaltar, que acá se identificó una alta resistencia local a la Ciprofloxacina, lo que se relaciona con los estudios previos mencionados y refuerza la tendencia a evitar el uso de quinolonas de forma empírica.

Frente a lo anterior no sorprende la alta tasa de resistencia a beta-lactámicos, incluso en asociación con inhibidores de betalactamasa. Las altas tasas de resistencia a Ceftriaxona en la institución (35.5\% para E.coli, $68.8 \%$ para $K$. pneumoniae). Esto sumado a la alta tasa de prescripción empírica de este antimicrobiano, invita a los encargados de diseñar los protocolos institucionales a replantear el uso de cefalosporinas de tercera generación como tratamiento empírico en pacientes con infección urinaria.

Por último, este trabajo encontró que casi la mitad de los pacientes había recibido antibioticoterapia previa, lo que refleja el uso excesivo de antimicrobianos, y esto aumenta el problema de la resistencia, que es más frecuente en países de ingresos bajos y medianos en los que las IVU constituyen una de las principales causas de automedicación antibiótica $(36,37)$. Se hace el llamado a la comunidad en general de evitar la automedicación, y a la comunidad médica de tener mejores pautas de prescripción.

Algunas de las limitaciones de este trabajo incluyen un número de muestra relativamente bajo en comparación con otros trabajos, así como el muestreo por conveniencia, el cual limita la representatividad de la muestra y la reproducibilidad del estudio.

Por último, las tasas de resistencia a antibióticos marcadores permiten inferir una baja prevalencia de espectro extendido, así como una alta prevalencia de $K$. pneumoniae con este perfil de resistencia. Sin embargo, se reconoce la limitación para caracterizar mejor esta población de microorganismos dado que en el formato de registro de datos no se incluyó esta información de forma discriminada.

\section{Conclusiones}

Los microorganismos más frecuentemente aislados fueron el $E$. coli y el $K$. peumoniae, que presentan alta resistencia a beta-lactámicos, resistencia intermedia a cefalosporinas de tercera generación y baja resistencia a los aminoglucósidos y los carbapenémicos.

Es importante entonces, a partir de estos resultados, que se realicen estudios con un mayor número de muestra seleccionada bajo muestreo probabilístico, incluyendo pacientes con diversas patologías infecciosas con el fin de definir protocolos institucionales ajustados a los perfiles de resistencia locales, además de desarrollar estrategias de educación en salud que permitan abordar esta problemática desde un enfoque más integral.

\section{Conflictos de interés}

Los autores declaran no tener conflicto de interés.

\section{Financiación}

El presente trabajo fue financiado con recursos asignados por convocatoria interna de la Universidad Autónoma de Bucaramanga - UNAB.

\section{Referencias}

1. Gupta K, Grigoryan L, Trautner B. Urinary Tract Infection. Ann Intern Med [Internet]. 6 de octubre de 2017 [citado 23 de junio de 2020]; Disponible en: https://www.acpjournals.org/doi/abs/10.7326/ AITC201710030

2. McLellan LK, Hunstad DA. Urinary Tract Infection: Pathogenesis and Outlook. Trends Mol Med. 1 de noviembre de 2016;22(11):946-57. https://doi. org/10.1016/j.molmed.2016.09.003

3. Foxman B. Urinary tract infection syndromes: occurrence, recurrence, bacteriology, risk factors, and disease burden. Infect Dis Clin North Am. marzo de 2014;28(1):1-13. viii. https://doi.org/10.1016/j. pop.2010.04.001

4. Villarraga JDA, Parra JDI, Diaz DA, Cardenas AM, Chavarriaga J, Godoy MP. Guía de práctica clínica de infección de vías urinarias en el adulto. Rev 
Urol Colomb Colomb Urol J. agosto https://doi. org/10.1016/j.idc.2013.09.003

5. Litza JA, Brill JR. Urinary tract infections. Prim Care. septiembrede2010;37(3):491-507, de2018;27(2):12631. https://doi.org/10.1055/s-0038-1660528

6. Orrego-Marin CP, Henao-Mejía CP, Cardona-Arias JA. Prevalencia de infección urinaria, uropatógenos y perfil de susceptibilidad antimicrobiana. 2014; 39:8.

7. Serna LFC, Guerrero CED, Bernal GB. Ministro de Salud y Protección Social. 64.

8. Fandiño YRM, Ochoa EYC, Guerra SAG, Niño DSF, Gómez JAU, Prieto AC. Caracterización clínica de infecciones de vías urinarias producidas por enterobacterias productoras de betalactamasas de espectro extendido en Duitama (Colombia), durante 2010-2015. Infectio [Internet]. 20 de enero de 2017 [citado 23 de junio de 2020];21(1). https://doi. org/10.22354/in.v21i1.636

9. Blanco VM, Maya JJ, Correa A, Perenguez M, Muñoz JS, Motoa G, et al. Prevalencia y factores de riesgo para infecciones del tracto urinario de inicio en la comunidad causadas por Escherichia coli productor de betalactamasas de espectro extendido en Colombia. Enferm Infecc Microbiol Clin. noviembre de 2016;34(9):559-65. https://doi.org/10.1016/j. eimc.2015.11.017

10. Gómez Escobar C, Plata Salazar M, Sejnaui J, Rico C, Vanegas B. Resistencia de la E.coli en urocultivos de pacientes con sospecha de infección urinaria intr y extra-hospitalaria en la Fundación Santa Fe de Bogotá. Urol Colomb. 2009;18(1):8.

11. Alviz-Amador A, Gamero-Tafur K, CaraballoMarimon R, Gamero-Tafur J. Prevalence of urinary tract infection, uropathogens and susceptibility profile in a hospital of cartagena-Colombia in 2016. Rev Fac Med. 2018;66(3):313-7. https://doi.org/10.15446/ revfacmed.v66n3.62601

12. Villalobos Andrea Patricia , Barrero Liliana Isabel RSM, Ovalle María Victoria VD. Vigilancia de infecciones asociadas a la atención en salud, resistencia bacteriana y consumo de antibióticos en hospitales de alta complejidad, Colombia, 2011 [Internet]. Biomédica. 2014 [citado 20 de julio de 2020]. p. 6780. https://doi.org/10.7705/biomedica.v34i0.1698

13. Pardo SS, Pabón PR, Bermudez D. Susceptibilidad microbiológica de los uropatógenos aislados en la comunidad en Colombia periodo 2009-2013. 2015;11.

14. Pitout JDD, Laupland KB. Extended-spectrum beta-lactamase-producing Enterobacteriaceae: an emerging public-health concern. Lancet Infect Dis. marzo de 2008;8(3):159-66. https://doi.org/10.1016/ $\underline{\text { S1473-3099(08)70041-0 }}$

15. Pineda-Posada M,Arias G, Suárez-Obando F, Bastidas A, Ávila-Cortés Y. Factores de riesgo para el desarrollo de infección de vías urinarias por microorganismos productores de betalactamasas de espectro extendido adquiridos en la comunidad, en dos hospitales de Bogotá D.C., Colombia. Infectio [Internet]. 10 de abril de 2017 [citado 23 de junio de 2020];21(3). https:// doi.org/10.22354/in.v21i3.670

16. Naber KG, Schito G, Botto H, Palou J, Mazzei T. Surveillance study in Europe and Brazil on clinical aspects and Antimicrobial Resistance Epidemiology in Females with Cystitis (ARESC): implications for empiric therapy. Eur Urol. noviembre de 2008;54(5):1164-75. https://doi.org/10.1016/j.eururo.2008.05.010

17. Walker E, Lyman A, Gupta K, Mahoney MV, Snyder GM, Hirsch EB. Clinical Management of an Increasing Threat: Outpatient Urinary Tract Infections Due to Multidrug-Resistant Uropathogens. Clin Infect Dis Off Publ Infect Dis Soc Am. 01 de 2016;63(7):960-5. https://doi.org/10.1093/cid/ciw396

18. Nocua-Báez LC, Cortés JA, Leal AL, Arias GF, Ovalle-Guerro MV, Saavedra-Rojas SY, et al. Antimicrobial susceptibility profile in urinary pathogens causing community-acquired infections in diabetic patients in Colombia. Biomédica. 1 de septiembre de 2017;37(3):353-60. https://doi. org/10.7705/biomedica.v37i3.3348

19. Nocua-Báez LC, Cortes-Luna JA, Leal-Castro AL, Arias-León GF, Ovalle-Guerro MV, SaavedraRojas SY, et al. Susceptibilidad antimicrobiana de enterobacterias identificadas en infección urinaria adquirida en la comunidad, en gestantes en nueve hospitales de Colombia. Rev Colomb Obstet Ginecol. 20 de diciembre de 2017;68(4):275-85. https://doi. org $/ 10.18597 / \mathrm{rcog} .928$

20. Akram M, Shahid M, Khan AU. Etiology and antibiotic resistance patterns of community-acquired urinary tract infections in J N M C Hospital Aligarh, India. Ann Clin Microbiol Antimicrob. 23 de marzo de 2007;6(1):4. https://doi.org/10.1186/1476-0711-6-4

21. Mohammed MA,AlnourTMS, Shakurfo OM,Aburass MM. Prevalence and antimicrobial resistance pattern of bacterial strains isolated from patients with urinary tract infection in Messalata Central Hospital, Libya. Asian Pac J Trop Med. 1 de agosto de 2016;9(8):7716. https://doi.org/10.1016/j.apjtm.2016.06.011

22. Pineda-Posada M, Arias G, Suárez-Obando F, Bastidas A, Ávila-Cortés Y. Factores de riesgo para el desarrollo de infección de vías urinarias por microorganismos productores de betalactamasas de espectro extendido adquiridos en la comunidad, en dos hospitales de Bogotá D.C., Colombia. Infectio. 2017;21(3):141-7. https://doi.org/10.22354/in.v21i3.670

23. Caicedo Pablo Santiago, Martínez M. Tatiana, Menéses D. Edgar, Joaqui Wilson Germán, Imbachí I. Richard, Mahe P. Diego A ER. Etiología y resistencia bacteriana en infección de vias urinarias en el Hospital Universitario San José de Popayán, Colombia entre 
33. Grodin L, Conigliaro A, Lee SY, Rose M, Sinert R. Comparison of UTI antibiograms stratified by ED patient disposition. Am J Emerg Med. 1 de septiembre de 2017;35(9):1269-75. https://doi.org/10.1016/j. ajem.2017.03.061

34. Li G, Zhao S, Wang S, Sun Y, Zhou Y, Pan X. A 7-year surveillance of the drug resistance in Klebsiella pneumoniae from a primary health care center. Ann Clin Microbiol Antimicrob. 9 de noviembre de 2019;18(1):34. https://doi.org/10.1186/s12941-019$\underline{0335-8}$

35. El Bouamri MC, Arsalane L, El Kamouni Y, Zouhair S. Antimicrobial susceptibility of urinary Klebsiella pneumoniae and the emergence of carbapenemresistant strains: A retrospective study from a university hospital in Morocco, North Africa. Afr J Urol. 1 de marzo de 2015;21(1):36-40. https://doi. org/10.1016/j.afju.2014.10.004

36. Ocan M, Obuku EA, Bwanga F, Akena D, Richard S, Ogwal-Okeng J, et al. Household antimicrobial selfmedication: a systematic review and meta-analysis of the burden, risk factors and outcomes in developing countries. BMC Public Health. 1 de diciembre de 2015;15(1):742. https://doi.org/10.1186/s12889-0152109-3

37. Torres NF, Chibi B, Middleton LE, Solomon VP, Mashamba-Thompson TP. Evidence of factors influencing self-medication with antibiotics in low and middle-income countries: a systematic scoping review. Vol. 168, Public Health. Elsevier B.V.; 2019. p. 92-101. https://doi.org/10.1016/j.puhe.2018.11.018

28. Urinary tract infection (lower): antimicrobial prescribing. Urin Tract Infect: 35.

29. Kang C-I, Kim J, Park DW, Kim B-N, Ha U-S, Lee S-J, et al. Clinical Practice Guidelines for the Antibiotic Treatment of Community-Acquired Urinary Tract Infections. Infect Chemother. 1 de marzo de 2018;50(1):67-100. https://doi.org/10.3947/ ic. 2018.50 .1 .67

30. Goodlet KJ, Benhalima FZ, Nailor MD. A Systematic Review of Single-Dose Aminoglycoside Therapy for Urinary Tract Infection: Is It Time To Resurrect an Old Strategy? Antimicrob Agents Chemother [Internet]. 1 de enero de 2019 [citado 4 de septiembre de 2020];63(1). https://doi.org/10.1128/AAC.02165-18

31. Schito GC, Naber KG, Botto H, Palou J, Mazzei T, Gualco L, et al. The ARESC study: an international survey on the antimicrobial resistance of pathogens involved in uncomplicated urinary tract infections. Int J Antimicrob Agents. noviembre de 2009;34(5):40713. https://doi.org/10.1016/j.ijantimicag.2009.04.012

32. Tandogdu Z, Wagenlehner FME. Global epidemiology of urinary tract infections. Vol. 29, Current Opinion in Infectious Diseases. Lippincott Williams and Wilkins; 2016. p. 73-9. https://doi.org/10.1097/QCO.0000000000000228 